\title{
MODELO DE EVALUACIÓN DE RESILIENCIA URBANA FRENTE A INUNDACIONES FLUVIALES \\ Papel potencial de los planes reguladores comunales en Chile
}

\author{
Assessment model of urban flood resilience \\ Potential role of communal regulating plans in Chile
}

\author{
González González, Luis Eduardo \\ Departamento de Planificación y Diseño Urbano, Universidad del Bío-Bío. gonzalezgleduardo@gmail.com \\ Baeriswyl Rada, Sergio \\ Departamento de Planificación y Diseño Urbano, Universidad del Bío-Bío. sbaeriswyl@ubiobio.cl \\ Zazo Moratalla, Ana \\ Departamento de Planificación y Diseño Urbano, Universidad del Bío-Bío. azazo@ubiobio.cl \\ Alvarado Aedo, Sebastian \\ Investigador independiente. seba88alva@gmail.com
}

\begin{abstract}
RESUMEN
El artículo expone los resultados de un nuevo modelo de evaluación de resiliencia urbana frente a inundaciones fluviales aplicado en una ciudad intermedia del Valle Central de Chile, Los Ángeles. A través de este se busca determinar el papel potencial de los Planes Reguladores Comunales para incrementar las capacidades de resiliencia. Para ello se hace una radiografía a su funcionamiento analizando 3 propiedades claves: redundancia de subsistemas, tiempo de aprendizaje tras cada evento y, capacidad de respuesta localizada. Los resultados proporcionan datos cuantitativos que permiten orientar de mejor manera la planificación urbana de escala comunal mediante acciones de urbanismo táctico donde el umbral de la resiliencia es determinado por el tiempo de recuperación del sistema; específicamente, la investigación identifica el número de semanas óptimo para lograr la recuperación del sistema desde la perspectiva de áreas de coberturas de los equipamientos críticos en escenarios de emergencia.
\end{abstract}

Palabras clave: Inundaciones fluviales, plan regulador comunal, resiliencia frente a inundaciones fluviales. Bloque temático: morfologías urbanas

\begin{abstract}
The article presents the results of a new assessment model of urban flood resilience applied in an intermediate city of the Central Valley of Chile, Los Ángeles. Through this the aim is to determine the potential role of the Communal Regulating Plans in order to increase resilience capacities. To do this, one test is made of its functioning through the analysis of 3 key properties: subsystem redundancy, learning time after each event and localized response capacity. The expected results are aimed at providing quantitative data to better guide community-scale urban planning through tactical urbanism actions where the threshold of resilience is determined by the recovery time of the analysed system; specifically, an optimal number of weeks is defined to achieve the recovery of the system according to the areas of coverage of the critical equipment.
\end{abstract}

Keywords: river floods, Communal Regulating Plans, urban flood resilience.

Topic: urban morphology 


\section{Introducción}

En los últimos 40 años han ocurrido una serie de inundaciones a nivel mundial asociadas al cambio climático global que han costado la vida de miles de personas, destruyendo equipamientos críticos para la ciudad y sus comunidades; siendo la mayoría de éstos desencadenados por eventos de precipitación extrema que aumentan sostenidamente año tras año (Dartmouth Flood Observatory, 2017). Al respecto, observatorios internacionales revelan que las inundaciones son el tercer peligro natural más dañino globalmente (Wilby \& Keenan, 2012) y Chile no es una excepción a este fenómeno; ya que las características geográficas y el poblamiento de las llanuras fluviales hacen al país vulnerable frente a este tipo de desastres.

En este contexto, las inundaciones fluviales recientes acontecidas en áreas urbanas planificadas del Valle Central parecen evidenciar que los Planes Reguladores Comunales (PRC's) no han sido herramientas suficientemente efectivas para incrementar las capacidades adaptativas de las ciudades intermedias frente al riesgo de inundación por desborde de cauces; aun cuando los criterios sobre riesgos naturales han estado siempre presentes en la doctrina de la planificación urbana tradicional chilena (Baeriswyl, 2014). Sin embargo, no existe información sistematizada que permita verificar tal hipótesis y tampoco se han aplicado herramientas para evaluar el desempeño de los PRC's. Por lo tanto, el objetivo general de este artículo es definir el papel potencial de éstos en la construcción de resiliencia urbana frente a inundaciones fluviales, para ello se utiliza un modelo de evaluación diseñado por los autores para testear si efectivamente estos instrumentos tienen un papel potencial o no, basado en Flood-IMPAT+ del Politécnico di Milano (Molinari, 2016). Con el objeto de aplicar el modelo diseñado se utiliza como caso de estudio a Los Ángeles en la Región del Bío-Bío, dado que esa ciudad constituye un caso notable de una urbe que a través de acciones tácticas ha logrado adaptarse al fenómeno de las inundaciones.

Específicamente, el modelo de evaluación se compone de dos dimensiones de análisis (ver Fig. 01): i) Territorial, y; ii) Comunitaria. La primera dimensión se desglosa de la siguiente manera: categoría redundancia de subsistemas, y 2 subcategorías: redundancia de equipamientos críticos y; redundancia de espacios abiertos. En relación con la segunda dimensión, se compone de las categorías tiempo de aprendizaje tras cada evento y capacidad de respuesta localizada. Los resultados esperados apuntan a proporcionar datos cuantitativos que permitan orientar de mejor manera la planificación urbana de escala comunal en Chile e incrementar las capacidades de resiliencia a través de lineamientos de urbanismo estratégico, entendido como la capacidad de adaptabilidad de un sistema para reorganizarse después de una perturbación (Van Veelen, 2016). Así, los PRC's tienen el potencial de preparar al sistema urbano antes de que ocurra un desastre y transformar tácticamente la configuración sistémica de éste después de ocurrida la perturbación (o desastre), transformando los ambientes urbanos desde una condición insegura a una de mayor seguridad.

\section{Resiliencia urbana frente $a$ inundaciones fluviales: propiedades claves}

Existe abundante literatura sobre el concepto de resiliencia desde la década de 1970, habiendo sido popularizado y utilizado tanto en el ámbito académico, discursos políticos como por planificadores urbanos con diversos enfoques (Meerow, Newell, \& Stults, 2016). Este concepto brinda la oportunidad de entender sistemas socio-ecológicos complejos y su relación con la gestión sustentable vinculada al cambio climático global y la formulación de estrategias adaptativas (Folke, 2006; Pickett, et Al, 2013). El origen de la resiliencia se puede rastrear en Holling (1973), quien la describió como la habilidad de un sistema para mantenerse funcionando y sobreponerse frente a las perturbaciones que lo afecten. Sin embargo, habiendo pasado más de 40 años desde la aparición de aquel novel concepto, aun no existe consenso sobre la definición concreta de éste y menos cómo medirlo; ya que las discusiones han sido principalmente de orden teórico y carentes de evidencia empírica sólida, conduciendo a un razonamiento circular y sesgado (United Nations Development Programme , 2016). En este artículo, la resiliencia será entendida desde el enfoque socio-ecológico que, de acuerdo con Meerow, Newell y Stults (2016), se define como la habilidad de un sistema urbano y todo su sistema constituyente para mantener o rápidamente retornar a las funciones deseadas tras enfrentar una o más 
perturbaciones, adaptándose al cambio y transformando sistemas con limitaciones en sistemas con capacidades adaptativas. En este contexto, Liao (2012) propone el concepto resiliencia frente a inundaciones fluviales, sosteniendo que para incrementarla deben potenciarse medidas no estructurales a través de un manejo eficiente de los instrumentos de planificación territorial y políticas públicas acertadas (Greiving, 2006). Así, concordando con los autores antes señalados existen 3 propiedades claves de la resiliencia urbana frente a inundaciones fluviales: i) redundancia de subsistemas; ii) tiempo de aprendizaje tras cada evento, y; iii) capacidad de respuesta localizada. Enfatizando cada uno de ellos en el factor tiempo de adaptación como elemento determinante de la resiliencia, lo que en la actualidad se denomina caminos hacia la resiliencia, o también, umbrales hacia la resiliencia (Chelleri, et Al, 2015; Van Veelen, 2016).

En específico, la redundancia de subsistemas da cuenta de la capacidad de respuesta funcional y de distribución espacial de aquellos equipamientos críticos para la ciudad, con objeto que ésta y sus subsistemas no colapsen. Para que esto ocurra, Liao (2012) sostiene que los subsistemas deben ser diversos y flexibles en aspectos, tales como, rutas de evacuación y áreas de seguridad. Por otra parte, la capacidad de respuesta localizada promueve el desarrollo de ciudades autoorganizadas donde los ciudadanos puedan rápidamente actuar para reducir daños materiales y perdidas económicas, imitando comportamientos adaptativos a través del fortalecimiento de las redes comunitarias. Finalmente, el tiempo de aprendizaje y ajuste tras cada inundación implica adquirir conocimiento específico luego de cada evento. En este contexto, los PRC's tienen el potencial de planificar tácticamente la morfología urbana colocando los acentos en la resiliencia frente a inundaciones a través de la redundancia de subsistemas y, luego en su proceso de formulación. No obstante lo anterior, pese a que la resiliencia se ha instalado como un concepto ampliamente utilizado la experiencia internacional demuestra que no existen herramientas que se hayan focalizado en desmenuzar su funcionamiento a través de indicadores y evaluarlos; por el contrario, la mayor divulgación tanto técnica y científica se ha focalizado en el diseño de estrategias para ciudades resilientes (Ilmola, 2016). Por lo anterior, la medición empírica del concepto es el aporte de este artículo al conocimiento.

\section{Metodología para determinar el papel potencial de los Planes Reguladores Comunales}

Experiencias internacionales en la medición de la resiliencia se han focalizado en caracterizar a los elementos constitutivos de ésta con la finalidad de elaborar estrategias para ciudades resilientes (IImola, 2016). Sin embargo, los antecedentes aquí proporcionados ratifican que dichas tendencias fomentan el desarrollo circular del conocimiento teórico (Meerow, Newell, \& Stults, 2016).

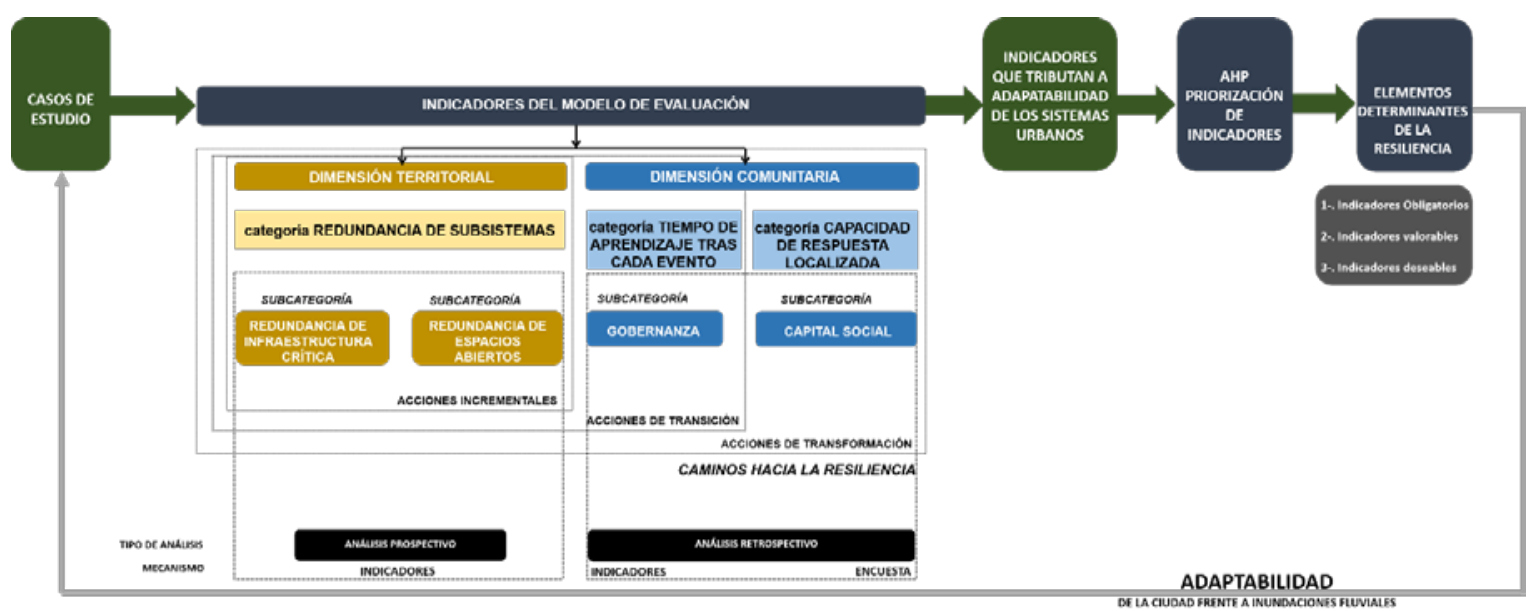

Fig. 01 Esquema metodológico-Fuente: elaboración propia 
El esquema metodológico (Fig. 01) consiste en el análisis prospectivo de un caso de estudio a través de 21 indicadores que se dividen en: dimensión territorial y dimensión comunitaria. En primer lugar, obteniendo resultados codificados por cada indicador, los que luego son ponderados mediante una priorización de análisis jerárquico (AHP). Finalmente, de acuerdo con la priorización realizada se distinguen indicadores de tipo: i) obligatorios; ii) valorables, y; iii) deseables en la concepción y modificación de un PRC para incrementar las capacidades de resiliencia de un sistema urbano.

\subsection{Modelo de evaluación de la de resiliencia frente a inundaciones fluviales y recolección de datos}

El modelo de evaluación se basa en Flood-IMPAT+ (Molinari, 2016) para hacer un análisis prospectivo de los PRC's. Éste aborda en detalle a la categoría redundancia de subsistemas a través de 8 indicadores desglosado en 2 subcategorías: redundancia de equipamientos críticos (6 indicadores) y; redundancia de espacios abiertos (2 indicadores). La ponderación de cada uno de ellos diferencia 5 niveles, donde: a mayor ponderación (del 1 al 5), existirá mayor capacidad de resiliencia instalada en la ciudad frente a inundaciones fluviales. Para ello se utiliza la Escala de Likert a través de la siguiente nomenclatura: 5 (muy alta); 4 (alta); 3 (medio); 2 (bajo), y; 1 (nula). En la Tabla 01 se detalla la codificación de cada uno de los rangos señalados de manera pormenorizada. En particular, los rangos de la subcategoría redundancia de equipamientos críticos y de la redundancia de espacios abiertos se definió en base a: i) estándares internacionales establecidos en Informes de la Cruz Roja Internacional (2014) y recomendaciones definidas por Flood-IMPAT+ (Molinari, 2016) y; ii) en cuanto al área de cobertura de cada equipamiento crítico se utilizaron rangos máximos de 15 minutos de desplazamiento a pie hacia cada lado del área desbordada por el cauce hídrico, propendiendo a la búsqueda de la integración de la morfología urbana y sus equipamientos. Es del caso señalar que se utiliza ese rango de tiempo considerando una velocidad promedio de $5,3 \mathrm{~km} / \mathrm{hr}$ de recorrido en condiciones ideales de una topografía que tiende a la horizontalidad. 


\begin{tabular}{|c|c|c|c|c|}
\hline $\begin{array}{c}\text { SUB- } \\
\text { CATEGORÍA }\end{array}$ & $\mathbf{N}^{\circ}$ & INDICADOR & CONDICIÓN & PUNTAJE \\
\hline \multirow[t]{6}{*}{$\begin{array}{l}\text { Redundancia } \\
\text { de } \\
\text { equipamientos } \\
\text { críticos }\end{array}$} & 1 & $\begin{array}{l}\text { Población viviendo en } \\
\text { área expuesta a una } \\
\text { amenaza (PVAEA) }\end{array}$ & $\begin{array}{ll}\text { - } & \text { - ó igual de } 200 \text { hab } \\
\text { - } & \text { hasta } 400 \text { hab } \\
\text { - } & \text { hasta } 600 \text { hab } \\
\text { - } & \text { hasta } 800 \text { hab } \\
\text { - } & \text { + de } 800 \text { hab }\end{array}$ & $\begin{array}{l}-5 \\
-\quad 4 \\
-\quad 3 \\
-\quad 2 \\
-\quad 1\end{array}$ \\
\hline & 2 & $\begin{array}{l}\text { Equipamientos críticos } \\
\text { emplazados en área } \\
\text { expuesta a una } \\
\text { amenaza (ECEAEA) }\end{array}$ & $\begin{array}{ll}\text { - } & 0 \\
\text { - } & \text { hasta } 1 \\
\text { - } & \text { hasta } 3 \\
\text { - } & \text { hasta } 5 \\
\text { - } & + \text { de } 5\end{array}$ & 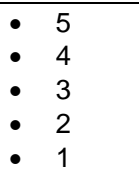 \\
\hline & 3 & $\begin{array}{l}\text { Edificios de } \\
\text { Administración Pública } \\
\text { emplazados en área } \\
\text { expuesta a una } \\
\text { amenaza (EAPEAEA) }\end{array}$ & $\begin{array}{ll}- & 0 \\
\text { - } & \text { hasta } 1 \\
\text { - } & \text { hasta } 3 \\
\text { - } & \text { hasta } 5 \\
\text { - } & + \text { de } 5\end{array}$ & $\begin{array}{ll} & 5 \\
- & 4 \\
- & 3 \\
- & 2 \\
- & 1\end{array}$ \\
\hline & 4 & $\begin{array}{l}\text { Cobertura de } \\
\text { equipamientos críticos } \\
\text { que van en ayuda de } \\
\text { la población en } \\
\text { escenarios de } \\
\text { emergencia } \\
\text { (CECVAP) }\end{array}$ & $\begin{array}{l}\text { - } \quad \text { Cobertura de + de } 1 \text { equipamiento en cada } \\
\text { ribera del cauce desbordado } \\
\text { - Cobertura de } 1 \text { equipamiento en cada ribera } \\
\text { del cauce desbordado } \\
\text { - Cobertura de } 1 \text { equipamiento en solo una } \\
\text { ribera del cauce desbordado } \\
\text { - Existencia de } 1 \text { equipamiento afuera del área } \\
\text { de cobertura } \\
\text { - Ausencia de equipamiento de éstas } \\
\text { características en la ciudad }\end{array}$ & $\begin{array}{ll} & 5 \\
- & 4 \\
- & 3 \\
- & 2 \\
- & 1\end{array}$ \\
\hline & 5 & $\begin{array}{l}\text { Cobertura de } \\
\text { Equipamientos críticos } \\
\text { que prestan ayuda de } \\
\text { atención específica en } \\
\text { escenarios de } \\
\text { emergencia } \\
\text { (CECPAAE) }\end{array}$ & $\begin{array}{l}\text { - Cobertura de + de } 1 \text { equipamiento en cada } \\
\text { ribera del cauce desbordado } \\
\text { - Cobertura de } 1 \text { equipamiento en cada ribera } \\
\text { del cauce desbordado } \\
\text { - Cobertura de } 1 \text { equipamiento en solo una } \\
\text { ribera del cauce desbordado } \\
\text { - Existencia de } 1 \text { equipamiento afuera del área } \\
\text { de cobertura } \\
\text { - Ausencia de equipamiento de éstas } \\
\text { características en la ciudad }\end{array}$ & $\begin{array}{ll} & 5 \\
\cdot & 4 \\
- & 3 \\
- & 2 \\
- & 1 \\
\end{array}$ \\
\hline & 6 & $\begin{array}{l}\text { Cobertura de } \\
\text { Equipamientos críticos } \\
\text { que prestan ayuda } \\
\text { multipropósito en } \\
\text { escenarios de } \\
\text { emergencia } \\
\text { (CECPAM) }\end{array}$ & $\begin{array}{l}\text { - Cobertura de + de } 1 \text { equipamiento en cada } \\
\text { ribera del cauce desbordado } \\
\text { - Cobertura de } 1 \text { equipamiento en cada ribera } \\
\text { del cauce desbordado } \\
\text { - Cobertura de } 1 \text { equipamiento en solo una } \\
\text { ribera del cauce desbordado } \\
\text { - Existencia de } 1 \text { equipamiento afuera del área } \\
\text { de cobertura } \\
\text { - Ausencia de equipamiento de éstas } \\
\text { características en la ciudad }\end{array}$ & $\begin{array}{ll}\cdot & 5 \\
\cdot & 4 \\
- & 3 \\
- & 2 \\
- & 1 \\
\end{array}$ \\
\hline \multirow[t]{2}{*}{$\begin{array}{l}\text { Redundancia } \\
\text { de espacios } \\
\text { abiertos }\end{array}$} & 7 & $\begin{array}{l}\text { Redundancia rutas de } \\
\text { evacuación (RRE) }\end{array}$ & $\begin{array}{ll}- & + \text { de } 3 \\
- & \text { hasta } 3 \\
- & \text { hasta } 2 \\
- & \text { hasta } 1 \\
- & 0 \\
\end{array}$ & $\begin{array}{ll} & 5 \\
\bullet & 4 \\
\bullet & 3 \\
- & 2 \\
- & 1 \\
\end{array}$ \\
\hline & 8 & $\begin{array}{l}\text { Redundancia áreas } \\
\text { seguras (RAS) }\end{array}$ & $\begin{array}{ll}\text { - } & \text { + de } 3 \\
\text { - } & \text { hasta } 3 \\
\text { - } & \text { hasta } 2 \\
\text { - } & \text { hasta } 1 \\
\text { - } & 0 \\
\end{array}$ & $\begin{array}{ll}- & 5 \\
\bullet & 4 \\
- & 3 \\
- & 2 \\
- & 1 \\
\end{array}$ \\
\hline
\end{tabular}

Tabla 01 Indicadores categoría redundancia de subsistemas-Fuente: elaboración propia

En la Tabla 02 se exponen los 8 indicadores de la dimensión comunitaria. Específicamente, la subcategoría gobernanza, construida a través de información obtenida luego de realizar entrevistas semi-estructuradas a informantes claves del gobierno local, realizada durante el mes de noviembre de 2018. 


\begin{tabular}{|c|c|c|c|c|}
\hline $\begin{array}{c}\text { SUB- } \\
\text { CATEGORÍA }\end{array}$ & $\mathbf{N}^{\circ}$ & INDICADOR & CONDICIÓN & PUNTAJE \\
\hline \multirow[t]{8}{*}{ Gobernanza } & 1 & $\begin{array}{l}\text { Gobernabilidad } \\
\text { (GOB) }\end{array}$ & $\begin{array}{ll}\text { - } & \text { Solo } 1 \\
\text { - } & \text { hasta } 2 \\
\text { - } & \text { hasta } 3 \\
\text { - } & \text { hasta } 4 \\
\text { - } & + \text { de } 4\end{array}$ & 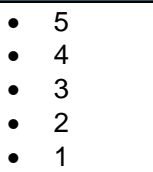 \\
\hline & 2 & $\begin{array}{l}\text { Institucionalidad } \\
\text { (INST) }\end{array}$ & $\begin{array}{ll}- & + \text { de } 3 \\
- & \text { hasta } 3 \\
\text { - } & \text { hasta } 2 \\
\text { - } & \text { hasta } 1 \\
\text { - } & \text { Ninguno }\end{array}$ & $\begin{array}{l}-5 \\
-\quad 4 \\
-\quad 3 \\
-\quad 2 \\
-\quad 1\end{array}$ \\
\hline & 3 & $\begin{array}{l}\text { Protocolo de acción y } \\
\text { educación de las } \\
\text { comunidades (PAEC) }\end{array}$ & $\begin{array}{ll}- & + \text { de } 3 \\
- & \text { hasta } 3 \\
- & \text { hasta } 2 \\
- & \text { hasta } 1 \\
- & \text { Ninguno }\end{array}$ & $\begin{array}{ll}- & 5 \\
- & 4 \\
- & 3 \\
- & 2 \\
- & 1 \\
\end{array}$ \\
\hline & 4 & $\begin{array}{l}\text { Implementación de } \\
\text { sistema de alerta } \\
\text { temprana (ISAT) }\end{array}$ & $\begin{array}{l}\text { - } \quad \text { Tiene y lo difunde más de } 2 \text { veces al año } \\
\text { - } \quad \text { Tiene y lo difunde } 2 \text { veces al año } \\
\text { - } \quad \text { Tiene pero lo difunde } 1 \text { vez al año } \\
\text { - } \quad \text { Tiene pero no lo ha difundido } \\
\text { - } \quad \text { No tiene sistema de alerta temprana } \\
\end{array}$ & $\begin{array}{ll} & 5 \\
- & 4 \\
- & 3 \\
- & 2 \\
- & 1 \\
\end{array}$ \\
\hline & 5 & $\begin{array}{l}\text { Evaluación post- } \\
\text { evento (EPE) }\end{array}$ & $\begin{array}{ll} & \text { de } 3 \\
\text { - } & \text { hasta } 3 \\
\text { - } & \text { hasta } 2 \\
\text { - } & \text { hasta } 1 \\
\text { - } & \text { Ninguna } \\
\end{array}$ & $\begin{array}{ll}- & 5 \\
- & 4 \\
- & 3 \\
- & 2 \\
- & 1 \\
\end{array}$ \\
\hline & 6 & $\begin{array}{l}\text { Focalización de la } \\
\text { inversión pública (FIP) }\end{array}$ & $\begin{array}{ll} & \text { - de } 3 \\
\text { - } & \text { hasta } 3 \\
\text { - } & \text { hasta } 2 \\
\text { - } & \text { hasta } 1 \\
& \text { Ninguna } \\
\end{array}$ & $\begin{array}{ll}\cdot & 5 \\
- & 4 \\
- & 3 \\
- & 2 \\
- & 1 \\
\end{array}$ \\
\hline & 7 & $\begin{array}{l}\text { Construcción de } \\
\text { mapas de } \\
\text { vulnerabilidad } \\
(\mathrm{CMV})\end{array}$ & $\begin{array}{l}\text { - } \quad \text { existe un mapa y este ha sido difundido } \\
\text { - } \quad \text { existe un mapa } \\
\text { - } \quad \text { se está elaborando el mapa } \\
\text { - } \quad \text { el mapa no está actualizado } \\
\text { - } \quad \text { no existe el mapa }\end{array}$ & $\begin{array}{ll}\cdot & 5 \\
- & 4 \\
- & 3 \\
- & 2 \\
- & 1 \\
\end{array}$ \\
\hline & 8 & $\begin{array}{l}\text { Construcción de } \\
\text { mapas de exposición } \\
\text { (CME) }\end{array}$ & $\begin{array}{l}\text { - } \quad \text { existe un mapa y este ha sido difundido } \\
\text { - } \quad \text { existe un mapa } \\
\text { - } \quad \text { se está elaborando el mapa } \\
\text { - } \quad \text { el mapa no está actualizado } \\
\text { - } \quad \text { no existe el mapa }\end{array}$ & $\begin{array}{ll}\cdot & 5 \\
\bullet & 4 \\
\bullet & 3 \\
- & 2 \\
- & 1 \\
\end{array}$ \\
\hline
\end{tabular}

Tabla 02 Indicadores subcategoría gobernanza-Fuente: elaboración propia

Finalmente, en la Tabla 03 se exponen 4 indicadores que también forman parte de la dimensión comunitaria. Específicamente, la subcategoría capital social que fue construida a través de una encuesta ${ }^{1}$ aplicada en las áreas que cuentan con antecedentes de inundaciones en Los Ángeles, realizada durante el mes de noviembre de 2018.

\footnotetext{
${ }^{1}$ La encuesta fue diseñada por los autores en colaboración con el Departamento de Estadísticas de la Universidad del Bío-Bío, siguiendo el correspondiente proceso de validación científica y un tamaño de muestra de 200 encuestas.
} 


\begin{tabular}{|c|c|c|c|c|}
\hline $\begin{array}{c}\text { SUB- } \\
\text { CATEGORÍA }\end{array}$ & $\mathbf{N}^{\circ}$ & INDICADOR & CONDICIÓN & PUNTAJE \\
\hline \multirow[t]{5}{*}{ Capital social } & 1 & $\begin{array}{l}\text { Fortalecimiento de las } \\
\text { relaciones sociales } \\
\text { (FRS) }\end{array}$ & $\begin{array}{ll}\text { - } & \text { + del } 50 \% \text { de los encuestados } \\
\text { - } & \text { hasta el } 50 \% \text { de los encuestados } \\
\text { - } & \text { hasta el } 25 \% \text { de los encuestados } \\
\text { - } & \text { hasta el } 10 \% \text { de los encuestados } \\
\text { - } & \text { - del } 10 \% \text { de los encuestados }\end{array}$ & $\begin{array}{l}- \\
- \\
-\end{array}$ \\
\hline & 2 & $\begin{array}{l}\text { Capacidad de } \\
\text { respuesta localizada } \\
(\mathrm{CRL})\end{array}$ & $\begin{array}{ll}\text { - } & \text { + del } 50 \% \text { de los encuestados } \\
\text { - } & \text { hasta el } 50 \% \text { de los encuestados } \\
\text { - } & \text { hasta el } 25 \% \text { de los encuestados } \\
\text { - } & \text { hasta el } 10 \% \text { de los encuestados } \\
\text { - } & \text { - del } 10 \% \text { de los encuestados }\end{array}$ & $\begin{array}{l}\cdot 5 \\
-\quad 4 \\
-\quad 3 \\
-\quad 2 \\
-\quad 1\end{array}$ \\
\hline & 3 & Apego al barrio (AB) & $\begin{array}{ll} & \text { - del } 50 \% \text { de los encuestados } \\
\text { - } & \text { hasta el } 50 \% \text { de los encuestados } \\
\text { - } & \text { hasta el } 25 \% \text { de los encuestados } \\
\text { - } & \text { hasta el } 10 \% \text { de los encuestados } \\
\text { - } & \text { - del } 10 \% \text { de los encuestados } \\
\end{array}$ & $\begin{array}{ll} & 5 \\
- & 4 \\
- & 3 \\
- & 2 \\
- & 1 \\
\end{array}$ \\
\hline & 4 & $\begin{array}{l}\text { Tiempo de } \\
\text { recuperación (TR) }\end{array}$ & $\begin{array}{l}\text { - } \quad \text { de } 2 \text { semanas } \\
\text { - } \quad \text { Al menos } 2 \text { semanas } \\
\text { - } \quad \text { Al menos } 6 \text { semanas } \\
\text { - } \quad \text { Al menos } 8 \text { semanas } \\
\text { - } \quad \text { + de } 8 \text { semanas } \\
\end{array}$ & $\begin{array}{l}-1 \\
-\quad 4 \\
-\quad 3 \\
-\quad 2 \\
-\quad 1 \\
\end{array}$ \\
\hline & 5 & $\begin{array}{l}\text { Aprendizaje continuo } \\
\text { de usos específicos } \\
\text { (ACUE) }\end{array}$ & $\begin{array}{l}\text { - } \quad \text { Equipamientos que van en ayuda } \\
\text { - } \quad \text { Equipamientos que prestan ayuda de atención } \\
\text { especifica } \\
\text { - } \quad \text { Equipamientos que prestan ayuda } \\
\text { multipropósito } \\
\text { - Vivienda unifamiliar } \\
\text { - Vivienda colectiva }\end{array}$ & $\begin{array}{ll} & 5 \\
\text { - } & 4 \\
- & 3 \\
- & 2 \\
- & 1\end{array}$ \\
\hline
\end{tabular}

Tabla 03 Indicadores subcategoría capital social. Fuente: elaboración propia 


\subsubsection{Descripción del caso de estudio}

La investigación toma como área de estudio el Valle Central de Chile ya que allí se han concentrado una serie de devastadoras inundaciones detonadas por eventos de precipitaciones intensas y de corta duración desde la década de 1980 en adelante asociadas al fenómeno de El Niño y luego al cambio climático global. Focalizándose el artículo en el caso de Los Ángeles, Región del Bío-Bío, dado que permite ejemplificar una planificación urbana táctica en respuesta al fenómeno antes descrito, que ha tenido sus mayores impactos en las décadas de 1990 y 2000 producto de desbordes del Estero Quilque en el centro de la ciudad, en una superficie de 73 Há.

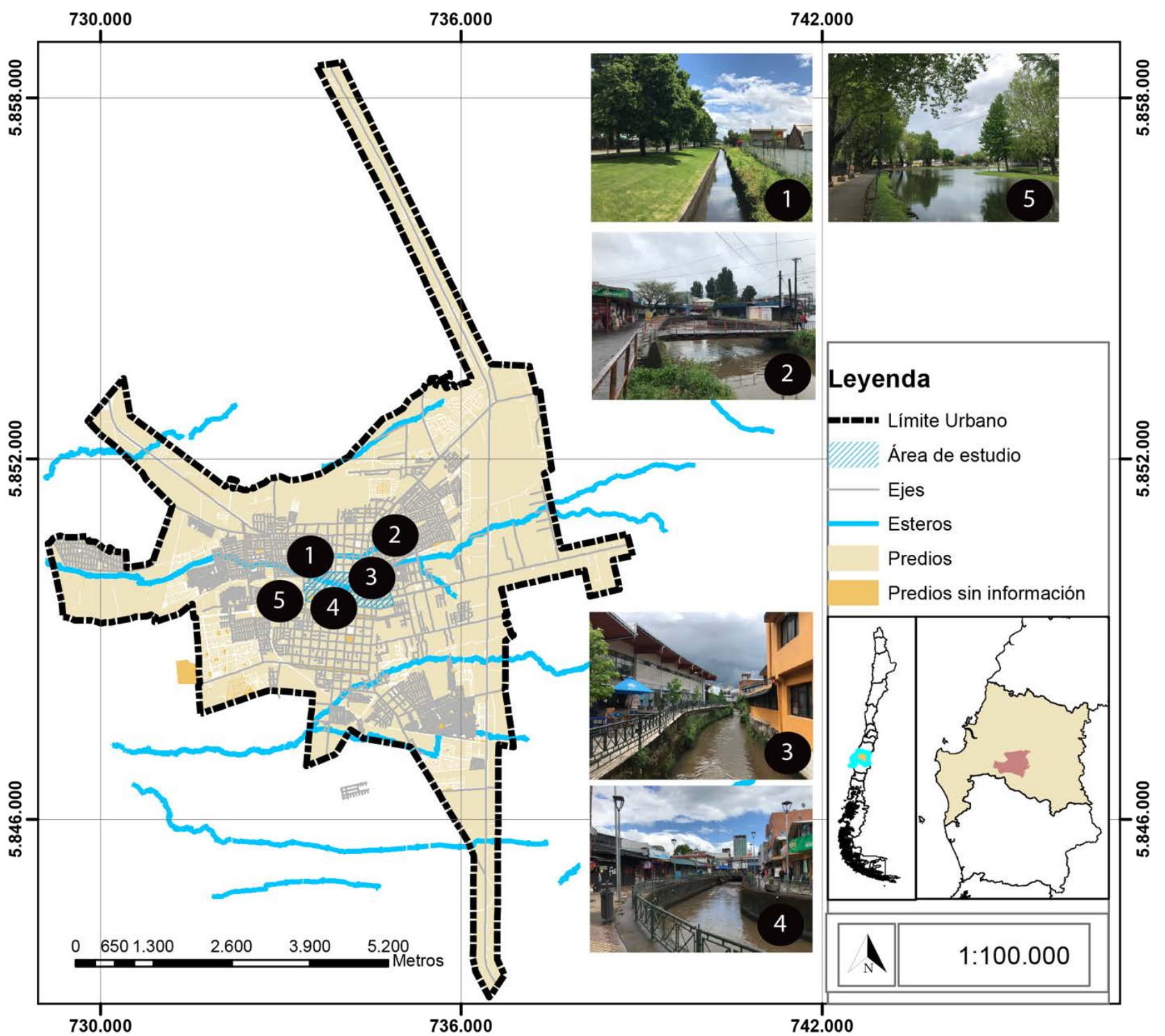

Mapa 01 Descripción del caso de estudio-Fuente: elaboración propia

En materia de planificación urbana reciente, la ciudad promulgó su primer PRC en 1991, reemplazándolo por otro en 2007, el cual se encuentra vigente. Particularmente, el área de estudio es parte del centro de la ciudad que es cruzada de oriente a poniente por el Estero Quilque, el cual según Mapa 01, imágenes 2, 3 y 4, es un cuerpo hídrico que se incorpora en el trazado de la ciudad dando lugar a usos mixtos (vivienda, equipamiento de servicios, educación y comercio), derivando este y otros cuerpos de agua menores en acequias y lagunas 
artificiales (imágenes 1 y 5 del mismo Mapa 01) que se desbordan de su cauce debido a fenómenos hidrometereológicos intensos. Complementariamente, en el Mapa 02 se muestra la zonificación establecida por el PRC vigente de la ciudad, donde se observa que asociado al Estero Quilque no existen definiciones de zonas de restricción o riesgo, las que en el Mapa 02, se grafican en gamas de rojos y naranjos.

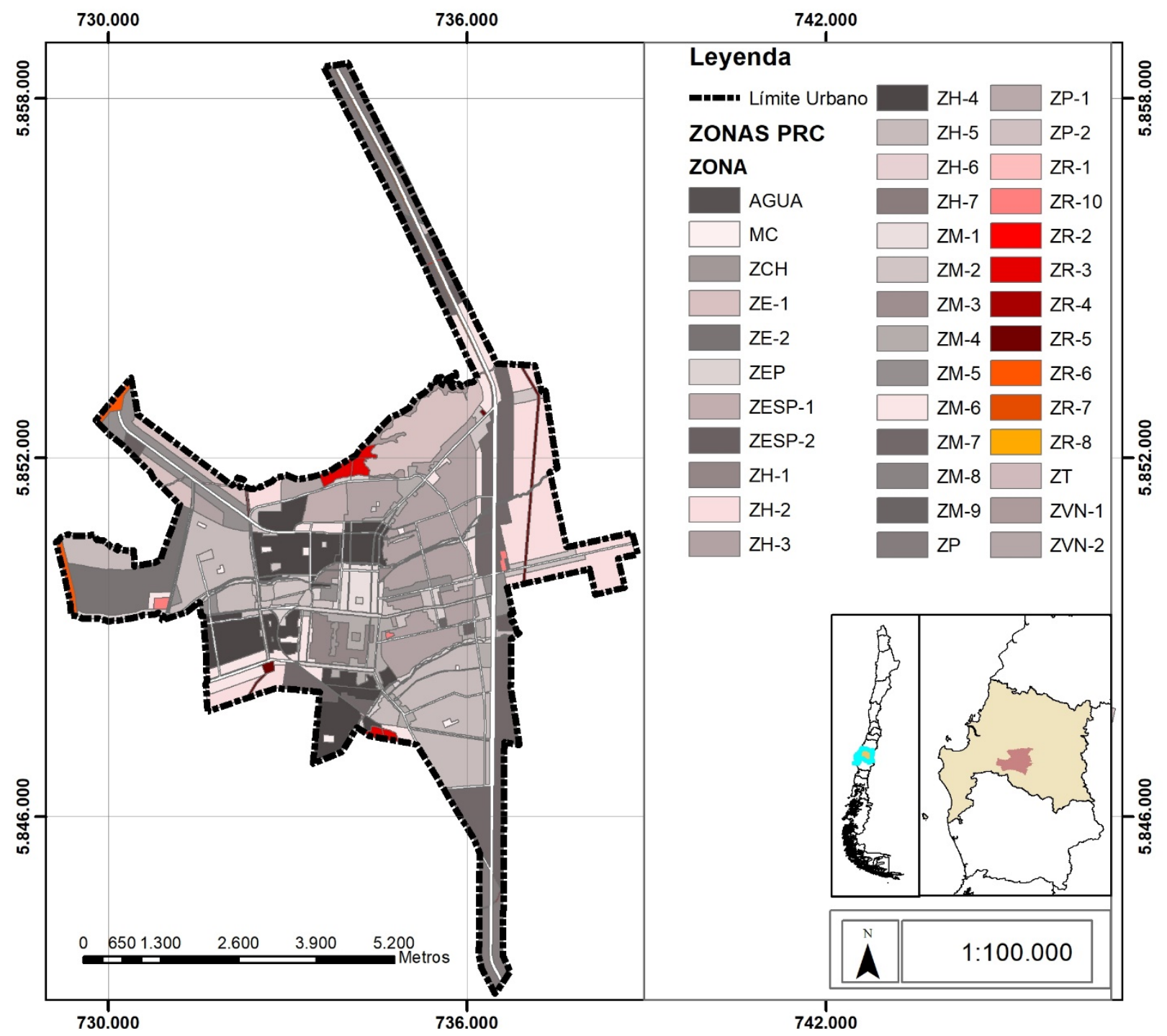

Mapa 02 Plan Regulador Comunal de Los Ángeles vigente-Fuente: elaboración propia (basado en IDE Chile)

Producto del Mapa 02 se observa que las restricciones de zonificación del PRC de la ciudad de Los Ángeles se localizan en áreas periféricas de esta; sin embargo, existen restricciones normativas propias de la zona central (bordes del Estero Quilque) que sí cuentan con limitaciones de uso, aspecto que no es abordado en este artículo que se enfoca en las potencialidades y oportunidades de los referidos instrumentos. Dichos aspectos serán revisados en el siguiente apartado.

\section{Análisis de los datos obtenidos}

A continuación, se exponen los principales resultados obtenidos de la aplicación del modelo en las subcategorías analizadas. 


\subsection{Subcategoría redundancia de equipamientos críticos}
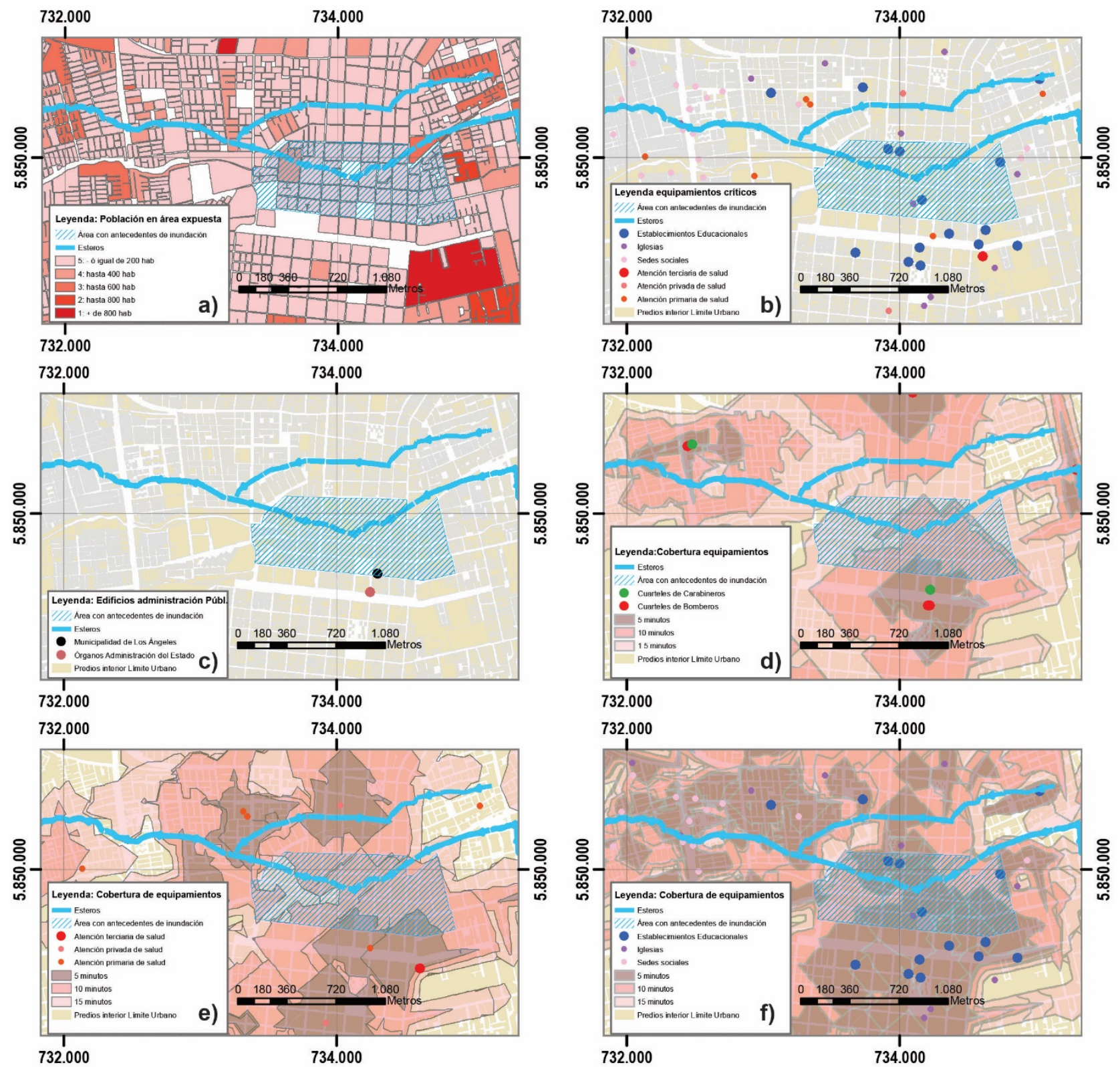

Mapa 03 cartografías asociadas a la subcategoría de equipamientos críticos-Fuente: elaboración propia

En primer lugar, en lo referente a la subcategoría redundancia de equipamientos críticos (ver Mapa 03), se observa que, en el análisis particularizado por indicador, en el área de estudio los resultados son los siguientes: i) en relación con la PVAEA (cartografía a), se trata de un área de la ciudad donde los usos existentes no fomentan la residencia, por lo tanto, la población permanente allí (CENSO, 2017) es baja en cada manzana y el puntaje en este indicador tiende a 5; ii) en cuanto a los ECEAEA (cartografía $b$ ) obtienen un bajo puntaje, 2 puntos, dado que al interior del polígono existen 5 equipamientos críticos, donde 4 de ellos corresponden a establecimientos educacionales y 1 iglesia; iii) en cuanto a los EAPEAEA (cartografía $c$ ) obtienen un puntaje de 4, dado que al interior del polígono únicamente se emplaza la Municipalidad de Los Ángeles; iv) en cuanto a la CECVAP (cartografía d) obtienen un puntaje de 3, dado que solo cuentan con equipamiento que va en ayuda de la comunidad en escenarios de emergencia y que se encuentren en un rango de 15 minutos 
caminando en una de sus riberas, 1 cuartel de carabineros y 1 cuartel de bomberos. En consecuencia, en escenarios adversos sólo una de las riberas del Estero Quilque podría ser atendido en un tiempo optimo; v) en cuanto a CECPAAE (cartografía e) obtiene un puntaje de 5 , debido a que en ambas riberas existen equipamientos que prestan ayuda de atención específica en escenarios de emergencia, principalmente compuesta por equipamientos de salud primaria y terciaria; vi) en cuanto a CECPAM (cartografía f) obtienen un puntaje de 4, dado que existen una serie de equipamientos que prestan ayuda multipropósito a las comunidades afuera del área de estudio (también expuesta al riesgo), tales como, establecimientos educacionales, iglesias y sedes sociales; sin embargo, dicha redundancia se concentra en la ribera sur, mientras que en la ribera norte solo existe 1 equipamiento con dichas características.

\subsection{Subcategoría redundancia de espacios abiertos}
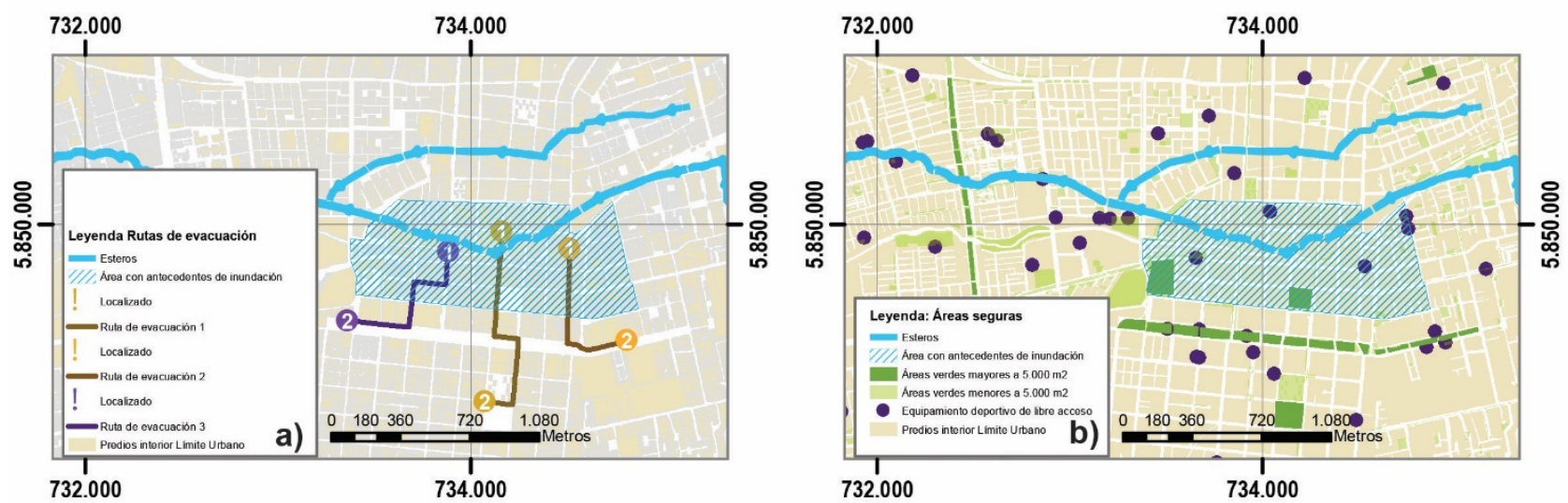

Mapa 04 cartografías asociadas a la subcategoría redundancia de espacios abiertos-Fuente: elaboración propia

En segundo lugar, en lo referente a subcategoría redundancia de espacios abiertos (ver Mapa 04), se observa que en el análisis pormenorizado se obtienen los siguientes resultados: vii) en cuanto a RRE (cartografía a) obtiene un puntaje de 5 , dado que la ciudad cuenta con una serie de potenciales redes viales que pueden ser utilizadas como rutas de evacuación, es decir más de 3 en cada ribera. Al respecto, solo con el propósito de ilustrar lo anterior se grafican 3 posibles alternativas pese a que actualmente no se encuentran reconocidas como tales; viii) en cuanto a RAS (cartografía $b$ ) obtiene un puntaje de 5 dado que existen una serie de áreas verdes sobre $5.000 \mathrm{~m} 2$ y equipamiento deportivo de libre acceso en ambas riberas, los cuales pueden funcionar como áreas seguras y de aprovisionamiento en escenarios de emergencia. 


\subsection{Subcategoría de gobernanza}

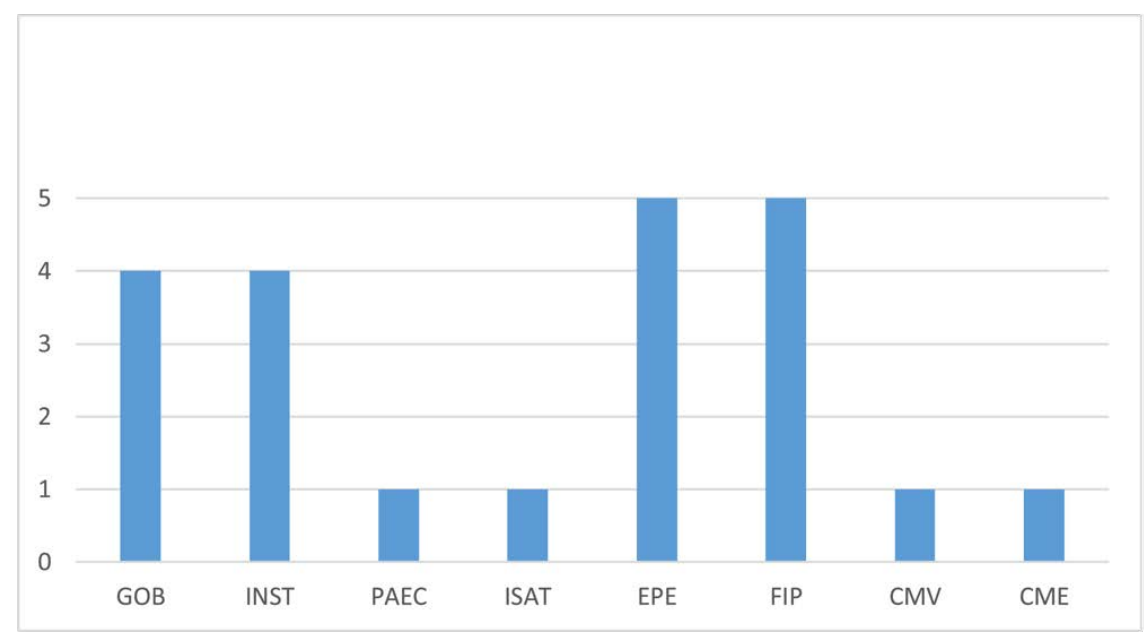

Fig. 02 Ponderaciones de subcategoría gobernanza-Fuente: elaboración propia

En lo referente a esta subcategoría (ver Fig. 02), se observa que en el análisis pormenorizado se obtienen los siguientes resultados: i) en cuanto a la GOB obtiene un puntaje de 4, dado que en escenarios de emergencia intervienen hasta dos entidades de gobierno durante el proceso de toma de decisiones; ii) en cuanto a la INST también obtiene un puntaje de 4 dado que se encuentran involucrados en la gestión del riesgo de desastre la Dirección de Ejecución de Proyectos y Servicios, la Oficina de Emergencia Municipal y la Dirección de Desarrollo Comunitario; iii) en cuanto a la PAEC se obtiene un puntaje de 1 dado que el gobierno local no cuenta con las instancias a las que este indicador refiere; iv) en cuanto a la ISAT también obtiene un puntaje de 1 dado que en la comuna no existen sistemas de alerta temprana; v) en cuanto a EPE se obtiene un puntaje de 5 dado que efectivamente el gobierno local ha desarrollado las instancias pertinentes de evaluación post evento; vi) en cuanto a la FIP obtiene un puntaje de 5 dado que el gobierno local ha priorizado inversión en las áreas inundables a través de mejoramiento y limpieza del cauce, así también, como inversión en la construcción de espacios públicos asociados a dichas áreas, obras que ya se han ejecutado y otras en cartera; vii) en cuanto a la CMV obtiene un puntaje de 1 dado que no existen mapas temáticos de vulnerabilidad frente a la amenaza de desborde de cauce; viii) igual situación ocurre con la CME que obtiene un puntaje de 1 al no existir mapas de exposición frente a la amenaza de inundación.

\subsection{Subcategoría de capital social}

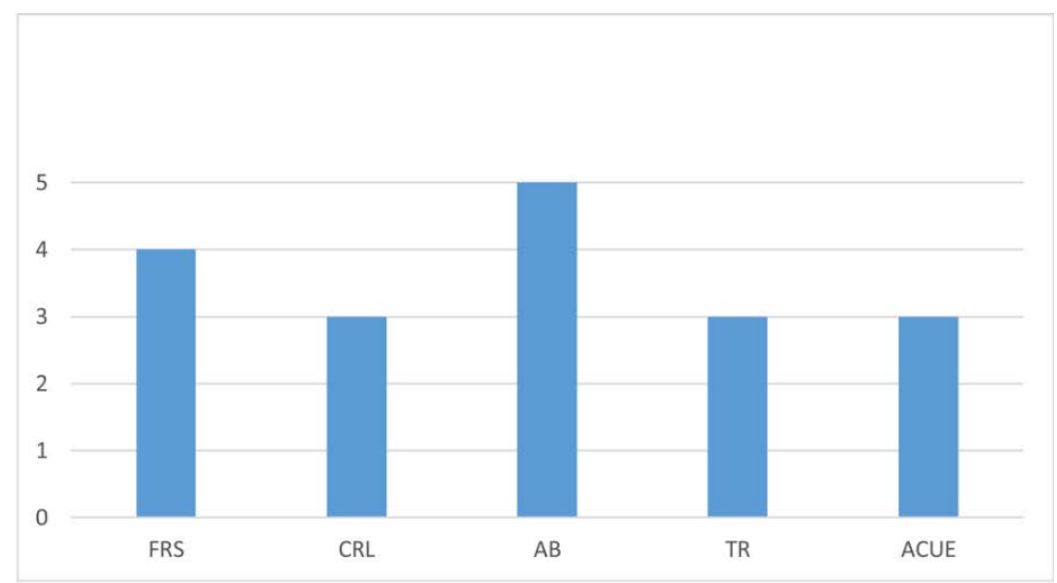

Fig. 03 Ponderaciones de subcategoría capital social-Fuente: elaboración propia 
En lo referente a esta subcategoría (ver Fig. 03), se observa que en el análisis pormenorizado se obtienen los siguientes resultados: i) en cuanto a FRS se obtiene un puntaje de 4 dado que la encuesta aplicada detectó que el $31 \%$ de la población participa activamente de organizaciones sociales; ii) en cuanto a la CRL se obtiene un puntaje de 3 dado que el $23 \%$ de los encuestados reconoció saber cómo actuar en escenarios de emergencia detonados por inundaciones, mientras que el $77 \%$ restante reconoció que sólo ha actuado en base a su instinto de supervivencia; iii) en cuanto a $A B$ se obtiene un puntaje de 5 dado que el $99 \%$ de los encuestados reconoció que nunca ha pensado en trasladarse a otro barrio de la ciudad pese a haber sido directamente afectado por una inundación y los motivos que explican aquello son la existencia de redes familiares y laborales en el área en que residen; iv) en cuanto al TR se obtiene un puntaje de 3 dado que el $52 \%$ de los encuestados reconoció que el tiempo de recuperación necesario después del desastre está en las 6 semanas, lo cual se explica en el tiempo necesario para retirar el agua del interior de las edificaciones, secado de la estructura y bienes de la población, para finalizar con la realización de la limpieza y orden de los muebles que componen los diversos recintos de la edificación; v) en cuanto a la ACUE se obtiene también un puntaje de 3 debido a que los encuestados sostienen que el equipamiento que presta ayuda de tipo multipropósito es aquella que más rápido se recupera luego de una perturbación como la analizada en este artículo.

\section{Discusión}

Desde el punto de vista de un análisis prospectivo, tanto el desempeño de la subcategoría redundancia de equipamientos críticos y redundancia de espacios abiertos logran altas ponderaciones lo cual se explica a partir de los siguientes criterios. Primero, en relación con la subcategoría redundancia de equipamientos críticos, un hecho fundamental es la apropiada ubicación y, por lo tanto, excelente cobertura que estos tienen en escenarios de emergencia, tanto aquellos que van en ayuda de las comunidades, tales como, los equipamientos de seguridad (bomberos y carabineros), como los que prestan ayuda de atención específica (salud primaria y terciaria) y aquellos equipamientos multipropósito que brindan acogida a los damnificados. Dicha redundancia es fundamental, debido a que si uno de ellos colapsa producto de la perturbación, existirá otro que pueda brindar el servicio y de esta manera alterar en menor medida la rutina de las personas. Es necesario precisar que los equipamientos que prestan ayuda multipropósito tienen una connotación especial; debido a que su participación durante el desastre es secuencial en función de su escala y según la propiedad del predio o inmueble, así en primera instancia los establecimientos educacionales son relevantes dado que actúan como albergues y centros de acopio para todo tipo de víveres, y también como centros logísticos para la prestación de ayuda; sin embargo, superados los días posteriores a la emergencia son las sedes sociales y los centros comunitarios religiosos los que adquieren ese rol dado que la demanda de espacio físico es menor y los establecimientos educacionales deben retomar sus actividades normales. Es del caso precisar que dadas las características del fenómeno analizado es primordial para que estos contribuyan a incrementar las capacidades de resiliencia, que la cobertura deberá ser optima en ambas riberas del cauce desbordado, de lo contrario, solo existirá una cobertura parcializada y la capacidad de resiliencia será menor.

Segundo, en relación con la subcategoría redundancia de espacios abiertos, esta obtiene el puntaje máximo dado que existe redundancia sistémica de posibles rutas de evacuación mediante recorridos a pie en escenarios de emergencia, al igual que, existe una amplia oferta de áreas verdes sobre $5.000 \mathrm{~m} 2$ que pueden actuar como áreas seguras (de reunión) en las primeras horas luego de la perturbación. En este contexto, la redundancia de subsistemas y de redes de espacios abiertos brinda la oportunidad de ser incorporados en los PRC's desde el punto de vista táctico, es decir, definir zonas de uso exclusivo para dicho tipo de equipamientos e incorporar las correspondientes declaratorias de utilidad pública de ensanches o aperturas de nuevas vías (vialidad estructurante de la ciudad) teniendo siempre el foco en optimizar las redes viales con objeto de disminuir el tiempo de respuesta luego de una emergencia, brindando las condiciones para facilitar la recuperación y perturbar en menor medida las rutinas de las personas damnificadas y del resto de la ciudad. Lo anterior, involucra un cambio de paradigma en el modo de hacer ciudad planificada; ya que hasta ahora lo 
que se busca es satisfacer demanda de usuarios mediante estudios de demanda de equipamientos y; por otro lado, responder a una rentabilidad social para que efectivamente puedan ser financiados y ejecutados.

Con respecto a la subcategoría gobernanza adquiere relevancia la focalización de inversión pública que tribute a la protección civil, lo cual puede ser recogido y validado durante las etapas tempranas de participación ciudadana con las organizaciones territoriales y con los Órganos de Administración del Estado a través de la evaluación ambiental estratégica a la que estos instrumentos deben someterse. No obstante, obtienen una baja ponderación elementos que debieran ser vitales en la gestión del riesgo de desastre y fortalecimiento de las comunidades a través de su educación en materia de riesgos y el conocimiento de su real situación, por lo tanto, pese a que su puntaje es bajo, los autores consideran que la elaboración y difusión de mapas de vulnerabilidad y exposición a determinadas amenazas es fundamental para crear conciencia sobre el contexto en el que cada una de las personas desarrolla sus rutinas.

Finalmente, con respecto a la subcategoría capital social, adquiere relevancia el fortalecimiento de las relaciones sociales y el apego al barrio por parte de las comunidades; dado que las personas que han consolidado sus redes familiares y laborales en una determinada área de la ciudad, aunque esta sea una área expuesta al riesgo, los resultados de la encuesta ratifican que dichas personas no estarían dispuestas a trasladarse áreas teóricamente más seguras, básicamente, por el apego al lugar.

En este contexto, de acuerdo con los antecedentes antes expuestos, los autores sostienen que existen un grupo de indicadores que presentan un alto potencial y, por lo tanto, debiera relevarse su consideración en distintas valoraciones. Estos son los pertenecientes a la subcategoría redundancia de subsistemas de tipo obligatoria; la subcategoría capital social valorable, y la subcategoría gobernabilidad deseable.

\section{Conclusiones}

Los resultados obtenidos tras la aplicación del modelo de evaluación sugieren una serie de recomendaciones para la elaboración de los PRC's en Chile y que estos además de responder a criterios normativos tradicionales de planificación urbana también puedan responder a criterios que buscan incrementar las capacidades de resiliencia de las ciudades intermedias en el Valle Central frente a inundaciones de tipo fluvial. Dichas recomendaciones siguen la siguiente estructura lógica. En primer lugar, estos instrumentos en sus condiciones actuales tienen el potencial técnico de definir las zonas de la ciudad donde debieran emplazarse los equipamientos críticos para el funcionamiento de esta y las redes viales para acceder a ellos, si bien esta facultad existe en los actuales PRC's lo que en este artículo es planteado requiere de un cambio de paradigma en el modo en que se hace ciudad en Chile, y por lo tanto, sean acciones tácticas que permitan enfrentar las perturbaciones a las que el sistema periódicamente debe enfrentar. Donde un requerimiento básico es contar con una óptima cobertura de equipamientos críticos (más de 1) a cada lado del cuerpo hídrico que presenta la amenaza de desborde, complementando a lo anterior, la cobertura de los mencionados equipamientos debe estar en el rango de 15 minutos de recorrido a pie; dado que esto brinda tiempo óptimo para que en escenarios de una crecida por eventos hidrometeorológicos puedan acceder al lugar los distintos servicios que van en ayuda de los damnificados y también que las personas puedan acceder a los equipamientos que prestan ayuda. Ocurriendo la misma situación con la red de espacios abiertos. Considerando lo anterior, la morfología urbana es entendida como el primer recurso que tienen las comunidades para proteger sus bienes y salvar sus vidas cuando se trata de una perturbación ocasionada por una inundación fluvial. Sin embargo, los resultados de este modelo de evaluación también sugieren que las características antes mencionadas son parte de las estrategias obligatorias en la ciudad ya que permiten resistir al evento, pero la gobernanza y capital social permiten incrementar las capacidades completas del sistema urbano y transformar áreas inseguras en otras de mayor seguridad. 


\section{BIBLOGRAFÍA}

BAERISWYL, S. (2014). Resiliencia urbana; aprender a habitar con las amenazas de la naturaleza. La experiencia del terremoto y tsunami de 2010 en las costas del Bío-Bío. Revista Márgenes (15), 11, 7-16.

INSTITUTO NACIONAL DE ESTADÍSTICAS, (2019), CENSO 2017, de www.ine.cl. Recuperado el 1 de marzo de 2019

CHELLERI, L., WATERS, J., OLAZABAL, M., \& MINUCCI, G. (2015). Resilience trade-offs: Addressing multiple scales a temporal aspect of urban resilience. Environment and Urbanization.

DARTMOUTH FLOOD OBSERVATORY. (2017). http://floodobservatory.colorado.edu/. Recuperado el 1 de septiembre de 2017, de http://floodobservatory.colorado.edu/: http://floodobservatory.colorado.edu/

FOLKE, C. (2006). Resilience: the emergence of a perspective for social-ecological systems analyses. Glob Environ Change, 16, 253-267.

GREIVING, S. (2006). Dealing with Natural Hazards in Germany's Planning Practice. En M. Fleischhauer, S. Greiving, \& S. Wanczura, Natural Hazards and Spatial Planning in Europe, 55-76.

HOLLING, C. (1973). Resilience and stability of ecological systems. Annual Review of Ecology and Systematics, 1-23.

ILMOLA, L. (2016). Approaches to measurement of urban resilience. In: Urban Resilience. A Transformative Approach. Eds. Yamagata, Y. \& Maruyama, H., pp. 207-237 Cham, Switzerland: Springer International Publishing. ISBN 978-3-319-39812-9

LIAO, K.-H. (2012). A Theory on Urban Resilience to Floods - A Basis for Alternative Planning Practices. Ecology and Society, 17(4), 15.

MEEROW, S., NEWELL, J., \& STULTS, M. (2016). Defining urban resilience: A review. Landscape and Urban Planning, 38-49.

MOLINARI, D. (2016). Flood-IMPAT+: an integrated Meso \& Micro scale procedure to assess territorial flood risk. Milano.

PICKETT, S., CADENASSO, M., \& MCGRATH, B. (2013). Resilience in ecology and urban design: Linking theory and practice for sustainable cities. En S. Pickett, M. Cadenasso, \& B. McGrath, Resilience in ecology and urban design: Linking theory and practice for sustainable cities. Dordrecht: Springer.

UNITED NATIONS DEVELOPMENT PROGRAMME. (2016). A new urban paradigm: pathways to sustainable development.

VAN VEELEN, P. (2016). Adaptive planning for resilient coastal waterfronts: Linking flood risk reduction with urban development in Rotterdam and New York City. Delft: A+BE | Architecture and the Built Environment.

WILBY, R. L., \& KEENAN, R. (2012). Adapting to flood risk under climate change. Progress in Physical Geography, 348-378. 